\title{
Candida albicans triggers the expression of inflammatory genes in human umbilical vein endothelial cells
}

\author{
JING YANG $^{1 *}$, WENLI FENG $^{1 *}$, JINYU ZHANG $^{1}$, ZHIQIN XI $^{1}$, YAN MA $^{1}$, \\ YANQING WANG $^{1}$, YING JI ${ }^{1}$, YAN WANG ${ }^{1}$ and WEI ZHANG $^{2}$ \\ ${ }^{1}$ Department of Dermatology and Venereology, The Second Hospital of Shanxi Medical University, Taiyuan, Shanxi 030001; \\ ${ }^{2}$ Department of Nephrology, Heji Hospital Affiliated to Changzhi Medical College, Changzhi, Shanxi 046011, P.R. China
}

Received March 18, 2015; Accepted April 21, 2016

DOI: $10.3892 /$ etm.2016.3464

\begin{abstract}
The aim of the present study was to investigate the pathogenesis of Candida albicans in human umbilical vein endothelial cells (HUVECs) and to screen for aberrantly expressed genes during the process of infection. GSE7355 (accession no.) was downloaded from the National Center of Biotechnology Information Gene Expression Omnibus database and used to identify the differentially-expressed genes (DEGs) between the two groups, which included 4 samples from an untreated HUVEC control group, and 4 samples from HUVECs exposed to C. albicans. Subsequently, the gene ontology (GO) function package was used to perform GO and pathway enrichment analysis, prior to the extraction of DEG correlations in the Kyoto Encyclopedia of Genes and Genomes. A protein-protein interaction (PPI) network was constructed using the String database. In total, 77 DEGs were identified, including 69 upregulated and 8 downregulated DEGs in the C. albicans-infected HUVEC samples. DEGs were significantly enriched in response to external stimuli and chemokine activity. In addition, DEG FBJ murine osteosarcoma viral oncogene homolog (FOS) and interleukin (IL)-6 were significantly enriched in the Toll-like receptor signaling pathway. Nuclear factor $\kappa$ light polypeptide gene enhancer in B cells 2 (NFKB2) was significantly enriched in the mitogen-activated protein kinase signaling pathway. In the interaction network of DEGs, according data included in the KEGG database, FOS
\end{abstract}

Correspondence to: Dr Wei Zhang, Department of Nephrology, Heji Hospital Affiliated to Changzhi Medical College, 271 Taihang East Road, Changzhi, Shanxi 046011, P.R. China E-mail: zzsidong@163.com

Dr Jing Yang, Department of Dermatology and Venereology, The Second Hospital of Shanxi Medical University, Taiyuan, Shanxi 030001, P.R. China

E-mail: yangj_y@126.com

*Contributed equally

Key words: Candida albicans, human umbilical vein endothelial cells, differentially-expressed genes, protein-protein interaction network and NFKB2 had higher connectivity degrees. Notably, FOS, IL-6 and intercellular adhesion molecule 1 were demonstrated to have higher connectivity degrees in the PPI network. FOS, IL-6 and NFKB2 may be important genes for $C$. albicans infection in HUVECs, and these genes may act as therapeutic targets to treat patients infected with $C$. albicans.

\section{Introduction}

The Candida species causes nosocomial bloodstream infections under certain circumstances (1). Candida albicans is a type of Candida species that may act as an opportunistic pathogen in immunocompromised or immunosuppressed patients (2). The incidence of candidemia is 1.1-24 cases/100,000 individuals, and the associated mortality is $>30 \%$, even when patients are treated with antifungal agents $(1,3,4)$. Therefore, elucidating the molecular mechanism underlying $C$. albicans infection and developing novel clinical approaches is urgently required.

In recent years, the mechanism underlying $C$. albicans infection has been clarified. Sun et al (5) demonstrated that Ssal plays a key role in the ability of $C$. albicans to damage host cells via binding to host endothelial cell cadherins and inducing host cell endocytosis in the models of oropharyngeal candidiasis. Furthermore, a previous study demonstrated that endothelial cells respond to infection with $C$. albicans by synthesizing interleukin (IL)-8 in vitro (6). Müller et al (7) suggested that activation of the p38 mitogen-activated protein kinase (MAPK) cascade is important for Candida-induced expression of chemokine (C-X-C Motif) ligand 8/IL-8 in endothelial cells. Several studies have demonstrated that the pathogenicity of $C$. albicans is induced by hyphae and epithelial cell damage $(8,9)$. Notably, Moyes et al $(10)$ demonstrated that the MAPK/MKP1/c-Fos signaling pathway is important for the formation of $C$. albicans hyphae in oral epithelial cells. However, the molecular mechanism underlying the host immune response and pathogen recognition is complex, and therefore our understanding of $C$. albicans infection is not fully complete.

Gene expression microarray analysis is used to observe changes in gene expression levels in various types of disease $(11,12)$. Müller et al $(7)$ provided the microarray data of GSE7355 (accession no.), and analyzed the differentially-expressed genes (DEGs) of human umbilical 
vein endothelial cells (HUVECs) following exposure to C. albicans. In addition, they investigated the nuclear factor $(\mathrm{NF})-\kappa \mathrm{B}$ and p38 MAPK signaling pathways in $C$. albicans infection. However, the interaction between DEGs was not analyzed, and a protein-protein interaction (PPI) network was not constructed.

To fully understand the HUVEC response to C.albicans, in the present study the microarray profile of HUVECs infected with $C$. albicans were analyzed and compared to a control. The DEGs between the two groups were screened, and a gene ontology (GO) function package was used to perform $\mathrm{GO}$ and pathway enrichment analysis of the DEGs. The extraction of the correlations among the DEGs were then carried out using the Kyoto Encyclopedia of Genes and Genomes (KEGG). Finally, a PPI network was constructed.

\section{Materials and methods}

Analysis of microarray data. The gene expression data GSE7355 (7) was downloaded from the National Center of Biotechnology Information Gene Expression Omnibus (http://www.ncbi.nlm.nih.gov/geo/) using the GPL96 platform of Affymetrix Human Genome U133A Arrays. A total of 8 samples were used in the present study, including 4 samples from untreated HUVEC monolayers (GSM177134, GSM177140, GSM17177141 and GSE177142) that served as the control group, and 4 samples from HUVECs exposed to C. albicans (GSM177136, GSM177137, GSM17177138 and GSE177139) that served as the experimental group. Raw data were downloaded for further analysis.

Data preprocessing and identification of DEGs. The Affy package (http://www.bioconductor.org/packages/rel ease/bioc/html/affy.html) (13) of Bioconductor (http://bioconductor.org/) was used to calculate the gene expression levels. Subsequently, a robust multiarray average algorithm (13) was used to perform the quartile data normalization. A t-test was carried out using the Limma package (http://www.bioconductor.org/packages/release/bioc/html/limma.html) (14) and applied to screen for DEGs between the two groups. $\mathrm{P}<0.05$ and $\log \mathrm{FCl}>0.58$ were selected as the criterion for DEGs.

GO and pathway enrichment analysis. Frequently, GO is used to conduct the functional enrichment analysis for large-scale genes (15). To identify the functions of the DEGs between the control and experimental samples, GO enrichment analysis was performed. In addition, KEGG pathway enrichment analysis was carried out for the DEGs, and bioinformatics databases containing all types of biochemistry signaling pathways were assessed (16). The GOFunction package (http://www.bioconductor.org/packages/release/bioc/html/GOFunction.html) of Bioconductor was used to perform the GO and pathway enrichment analysis. $\mathrm{A} P<0.05$ and gene counts $\geq 2$ were considered as the cut-off value. Furthermore, the correlation among DEGs was extracted according to the interactions of the genes in the KEGG.

Construction of a protein-protein interaction (PPI) network. The Search Tool for the Retrieval of Interacting Genes (STRING) database (http://string-db.org/) (17) is an online database that provides information on the interaction between proteins. In the present study, the STRING database was used to screen functional interactions between DEGs. A combined score $>4$ were regarded as the threshold. According to the criterion, Cytoscape (http://cytoscapeweb.cytoscape.org/) (18) was then used to display the PPI network.

\section{Results}

Identification of DEGs. Compared with the untreated HUVEC samples, a total of 77 DEGs were identified, including 69 upregulated DEGs corresponding to 187 transcripts, and 8 downregulated DEGs corresponding to 16 transcripts in the candida-infected HUVEC samples. The cluster heat map of 77 DEGs is presented in Fig. 1.

GO and pathway enrichment analysis. The GOfunction package was used to identify GO functions and signaling pathways for the significant DEGs. Notably, DEGs from the Candida-infected HUVEC samples were significantly enriched in response to external biological process stimuli $(\mathrm{P}<1.00 \mathrm{E}-15)$, chemokine activity $(\mathrm{P}=5.58 \mathrm{E}-08)$ and cytokine activity of molecular function ( $\mathrm{P}=4.17 \mathrm{E}-07$; Table I). In addition, DEGs were significantly enriched in the nodulation-like receptor signaling pathway $(\mathrm{P}=1.82 \mathrm{E}-08)$, toll-like receptor signaling pathway $(\mathrm{P}=1.00 \mathrm{E}-02)$ and MAPK signaling pathway $(\mathrm{P}=2.30 \mathrm{E}-02)$. The results of the pathway enrichment analysis are displayed in Table II.

The interaction network between the DEGs extracted from the KEGG, including 12 nodes and 10 edges, is shown in Fig. 2. In this network, FBJ murine osteosarcoma viral oncogene homolog (FOS) and nuclear factor $\kappa$ light polypeptide gene enhancer in B cells 2 (NFKB2; p49/p100) had the highest connectivity degrees.

Construction of the PPI network. In the present study, 52 nodes and 226 edges were used to construct the PPI network (Fig. 3). Notably, several nodes exhibited higher connectivity degrees: FOS (degree, 30), IL-6 (degree, 26), intercellular adhesion molecule 1 (degree, 22) and prostaglandin-endoperoxide synthase 2 (prostaglandin $\mathrm{G} / \mathrm{H}$ synthase and cyclooxygenase; degree, 21).

\section{Discussion}

The present study examined the gene expression data GSE7355 and investigated the HUVEC reaction patterns to the fungal pathogen $C$. albicans. In total, 77 DEGs were identified, including 69 upregulated DEGs corresponding to 187 transcripts and 8 downregulated DEGs corresponding to 16 transcripts in $C$. albicans-infected HUVEC samples. Notably, DEGs such as FOS and IL-6 were significantly enriched in the toll-like receptor signaling pathway, whereas NFKB2 was significantly enriched in the MAPK signaling pathway. In addition, FOS and IL-6 exhibited high connectivity degrees in the PPI network.

Toll-like receptors are the primary innate recognition system for microbial invaders in vertebrates, and are responsible for the immune response to microbial pathogens (19). Accumulating evidence suggests that the activation of toll-like 
Table I. Gene ontology functional enrichment analysis of DEGs.

\begin{tabular}{|c|c|c|c|c|}
\hline Gene ontology & Function & Total genes (n) & Enriched DEGs (n) & P-value \\
\hline \multirow[t]{5}{*}{ BP } & Response to external stimulus & 1,941 & 41 & $1.00 \mathrm{E}-15$ \\
\hline & Response to stress & 3,341 & 45 & 7.33E-15 \\
\hline & Cell migration & 1,015 & 26 & $5.02 \mathrm{E}-14$ \\
\hline & Response to stimulus & 7,662 & 63 & $2.77 \mathrm{E}-13$ \\
\hline & Cell motility & 1,095 & 26 & $2.94 \mathrm{E}-13$ \\
\hline \multirow[t]{5}{*}{$\mathrm{CC}$} & Extracellular space & 1,212 & 19 & 2.67E-07 \\
\hline & $\mathrm{I}-\kappa \mathrm{B} / \mathrm{NF}-\kappa \mathrm{B}$ complex & 5 & 3 & $6.61 \mathrm{E}-07$ \\
\hline & Bcl-3/NF-кB2 complex & 2 & 2 & $1.66 \mathrm{E}-05$ \\
\hline & Side of membrane & 300 & 8 & $3.10 \mathrm{E}-05$ \\
\hline & Cell surface & 645 & 10 & $2.90 \mathrm{E}-04$ \\
\hline \multirow[t]{5}{*}{$\mathrm{MF}$} & Protein binding & 8,384 & 61 & $5.89 \mathrm{E}-09$ \\
\hline & Chemokine activity & 46 & 6 & $5.58 \mathrm{E}-08$ \\
\hline & Chemokine receptor binding & 56 & 6 & $1.87 \mathrm{E}-07$ \\
\hline & Cytokine activity & 210 & 9 & 4.17E-07 \\
\hline & Binding & 12,580 & 70 & $2.53 \mathrm{E}-06$ \\
\hline
\end{tabular}

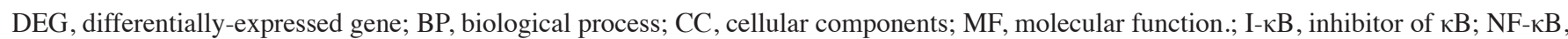
nuclear factor $\kappa \mathrm{B}$; Bcl-3, B cell lymphoma 3 .

Table II. KEGG signaling pathway analysis of DEGs.

\begin{tabular}{|c|c|c|c|}
\hline Name & Total genes $(\mathrm{n})$ & Enriched DEGs (n) & P-value \\
\hline NOD-like receptor signaling pathway & 58 & 8 & $1.82 \mathrm{E}-08$ \\
\hline Rheumatoid arthritis & 91 & 8 & $6.56 \mathrm{E}-07$ \\
\hline Malaria & 51 & 6 & 3.35E-06 \\
\hline Cytokine-cytokine receptor interaction & 265 & 11 & $8.15 \mathrm{E}-06$ \\
\hline Osteoclast differentiation & 128 & 7 & 7.98E-05 \\
\hline African trypanosomiasis & 35 & 4 & $1.86 \mathrm{E}-04$ \\
\hline Pathways in cancer & 326 & 10 & 2.87E-04 \\
\hline Chemokine signaling pathway & 189 & 7 & 0.000877 \\
\hline Chagas disease (American trypanosomiasis) & 104 & 5 & 0.001629 \\
\hline Epithelial cell signaling in Helicobacter pylori infection & 68 & 4 & 0.00236 \\
\hline Toll-like receptor signaling pathway & 102 & 4 & 0.010011 \\
\hline Amoebiasis & 106 & 4 & 0.011421 \\
\hline RIG-I-like receptor signaling pathway & 71 & 3 & 0.021015 \\
\hline Leishmaniasis & 72 & 3 & 0.021802 \\
\hline MAPK signaling pathway & 268 & 6 & 0.023041 \\
\hline B cell receptor signaling pathway & 75 & 3 & 0.02426 \\
\hline Small cell lung cancer & 85 & 3 & 0.033497 \\
\hline Bladder cancer & 42 & 2 & 0.047575 \\
\hline
\end{tabular}

KEGG, Kyoto Encyclopedia of Genes and Genomes; DEG, differentially-expressed genes; NOD, nodulation; RIG, retinoid acid-inducible gene; MAPK, mitogen-activated protein kinase.

receptors is important for Candida infection $(20,21)$. Notably, Zakikhany et al (22) suggested that hypha formation was crucial for the pathogenicity of $C$. albicans as well as the proinflammatory responses of mucosal tissues, which protect against subsequent fungal infection mediated by toll-like receptor 4 signaling (23). In the current study, FOS and IL-6 were significantly enriched in the toll-like receptor signaling pathway. FOS activation is mediated by hypha-associated surface moieties including MKP1 (24). Previously, a study demonstrated that hypha formation is dependent on the MAPK 


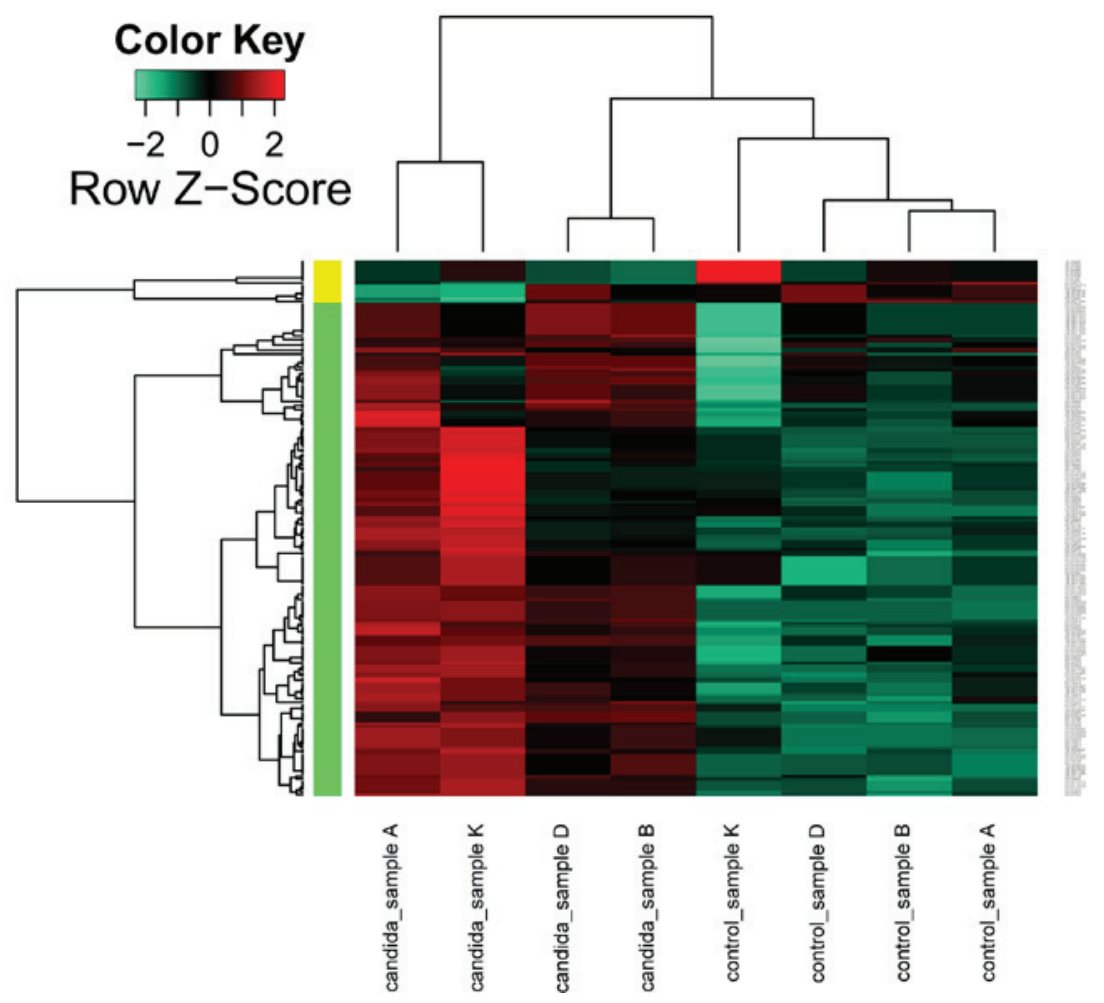

Figure 1. Cluster heat map of the 77 differentially expressed genes. The color represents the levels of gene expression in the 8 samples. The horizontal axis represents the samples, and the vertical axis represents the differentially expressed genes. Genes with high expression levels are presented in red; genes with low expression levels are presented in green.
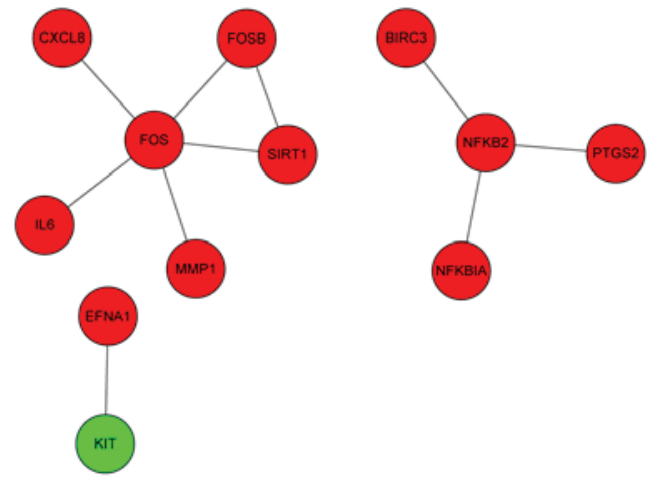

Figure 2. Interaction network of differentially expressed genes. Genes that are upregulated are presented in red; genes that are downregulated are presented in green.

response, constituted by the activation of FOS and MKP1 (8). Moyes et al (10) reported that the MAPK/MKP1/FOS signaling pathway is important for the formation of $C$. albicans hyphae in oral epithelial cells. Furthermore, the results of the present study demonstrated that FOS was upregulated, results which were concordant with those of Moyes et al (25) who demonstrated that FOS expression levels gradually increased with the progression of $C$. albicans infection in vaginal epithelial cells. In addition, hypha formation dependent on FOS activation and cell damage can induce the production of cytokines (10). IL-6 has been reported to protect against Candida infection (26). The present study demonstrated that IL-6 expression levels were upregulated, results which were concordant with those of Mostefaoui et al (27). Mostefaoui et al (27) demonstrated

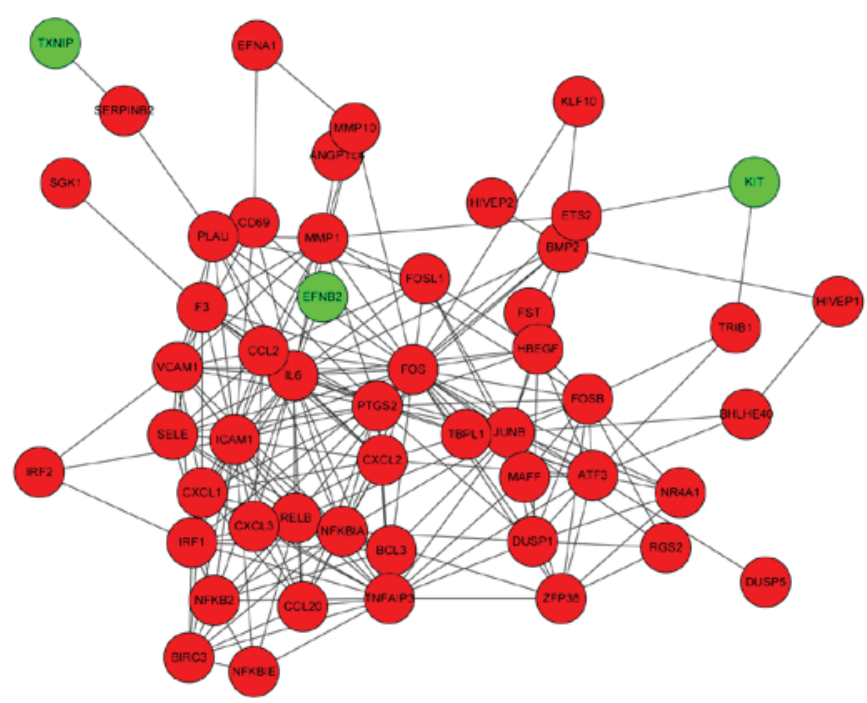

Figure 3. Protein-protein interaction network of differentially expressed genes. Genes that are upregulated are presented in red; genes that are downregulated are presented in green.

that IL-6 mRNA expression levels were significantly upregulated in human oral mucosa tissue following infection with C. albicans. These findings demonstrate that FOS and IL-6 may have important roles in $C$. albicans infection via the toll-like receptor signaling pathway.

Infection of epithelial cells with $C$. albicans causes the activation of NF- $\mathrm{KB}$, as well as a MAPK signaling response, which further induces a pro-inflammatory response (28). Furthermore, a previous study suggested that MAPK signaling 
pathways may served as targets for antifungal therapy (29). Notably, another investigation demonstrated that the MAPK signaling pathway enables human epithelial tissues to regulate innate immune responses against the hyphae of C. albicans (25). In the present study, NFKB2 expression levels were demonstrated to be upregulated and significantly enriched in the MAPK signaling pathway. NFKB2 is a member of the NF- $\kappa \mathrm{B}$ signal transduction pathway which has important roles in inflammatory and immune responses (30). Furthermore, fungal infection may be responsible for the release of chemotactic molecules in innate immune effector cells (31). Therefore, these data suggested that NFKB2 may have important roles in $C$. albicans infection via the MAPK signaling pathway.

In conclusion, the results of the present study further elucidated the mechanism underlying the effects of $C$. albicans infection in HUVECs. The screened DEGs, including FOS, IL-6 and NFKB2 may be important genes for the pathogenesis of $C$. albicans infection in HUVECs, and these genes may serve as therapeutic targets to treat patients infected with C. albicans. However, this study presented some limitations. The most important limitation was that the study was conducted using bioinformatics methods, but the results have not been further demonstrated through experiments. Therefore, further investigation using animal experiments should be considered.

\section{Acknowledgements}

The present study was supported by the Youth Science and Technology Research Foundation of Shanxi (grant no. 2012021035-3) and the Natural Science Foundation of Shanxi (grant no. 2012011045-3).

\section{References}

1. Wisplinghoff H, Bischoff T, Tallent SM, Seifert H, Wenzel RP and Edmond MB: Nosocomial bloodstream infections in US hospitals: Analysis of 24, 179 cases from a prospective nationwide surveillance study. Clin Infect Dis 39: 309-317, 2004.

2. Alby K, Schaefer D and Bennett RJ: Homothallic and heterothallic mating in the opportunistic pathogen Candida albicans. Nature 460: 890-893, 2009

3. Gudlaugsson O, Gillespie S, Lee K, Vande Berg J, Hu J, Messer S, Herwaldt L, Pfaller M and Diekema D: Attributable mortality of nosocomial candidemia, revisited. Clin Infect Dis 37: 1172-1177, 2003.

4. Stevens DA: Combination immunotherapy and antifungal chemotherapy. Clin Infect Dis 26: 1266-1269, 1998.

5. Sun JN, Solis NV, Phan QT, Bajwa JS, Kashleva H, Thompson A, Liu Y, Dongari-Bagtzoglou A, Edgerton M and Filler SG: Host cell invasion and virulence mediated by Candida albicans Ssa1. PLoS Pathog 6: e1001181, 2010.

6. Orozco AS, Zhou X and Filler SG: Mechanisms of the proinflammatory response of endothelial cells to Candida albicans infection. Infect Immun 68: 1134-1141, 2000.

7. Müller V, Viemann D, Schmidt M, Endres N, Ludwig S, Leverkus M, Roth J and Goebeler M: Candida albicans triggers activation of distinct signaling pathways to establish a proinflammatory gene expression program in primary human endothelial cells. J Immunol 179: 8435-8445, 2007.

8. Moyes DL, Runglall M, Murciano C, Shen C, Nayar D, Thavaraj S, Kohli A, Islam A, Mora-Montes H, Challacombe SJ and Naglik JR: A biphasic innate immune MAPK response discriminates between the yeast and hyphal forms of Candida albicans in epithelial cells. Cell Host Microbe 8: 225-235, 2010.

9. Jayatilake J, Samaranayake L, Lu Q and Jin L: IL-1alpha, IL-1ra and IL- 8 are differentially induced by Candida in experimental oral candidiasis. Oral Dis 13: 426-433, 2007.
10. Moyes DL, Murciano C, Runglall M, Kohli A, Islam A and Naglik JR: Activation of MAPK/c-Fos induced responses in oral epithelial cells is specific to Candida albicans and Candida dubliniensis hyphae. Med Microbiol Immunol 201: 93-101, 2012.

11. Sørlie T, Perou CM, Tibshirani R, Aas T, Johnsen H, Hastie T, Eisen MB, van de Rijn M, Jeffrey SS, Thorsen T, et al: Gene expression patterns of breast carcinomas distinguish tumor subclasses with clinical implications. Proc Natl Acad Sci USA 98: 10869-10874, 2001.

12. Beer DG, Kardia SL, Huang CC, Giordano TJ, Levin AM, Misek DE, Lin L, Chen G, Gharib TG, Thomas DG, et al: Gene-expression profiles predict survival of patients with lung adenocarcinoma. Nat Med 8: 816-824, 2002.

13. Gautier L, Cope L, Bolstad BM and Irizarry RA: Affy-analysis of Affymetrix GeneChip data at the probe level. Bioinformatics 20: 307-315, 2004.

14. Ritchie ME, Phipson B, Wu D, Hu Y, Law CW, Shi W and Smyth GK: Limma powers differential expression analyses for RNA-sequencing and microarray studies. Nucleic Acids Res 43: e47, 2015.

15. Ashburner M, Ball CA, Blake JA, Botstein D, Butler H, Cherry JM, Davis AP, Dolinski K, Dwight SS, Eppig JT, et al: Gene ontology: Tool for the unification of biology. Nat Genet 25: 25-29, 2000.

16. Kanehisa M and Goto S: KEGG: Kyoto encyclopedia of genes and genomes. Nucleic Acids Res 28: 27-30, 2000.

17. Franceschini A, Szklarczyk D, Frankild S, Kuhn M, Simonovic M, Roth A, Lin J, Minguez P, Bork P, von Mering C and Jensen LJ: STRING v9. 1: Protein-protein interaction networks, with increased coverage and integration. Nucleic Acids Res 41: D808-D815 (Database Issue), 2013.

18. Smoot ME, Ono K, Ruscheinski J, Wang PL and Ideker T: Cytoscape 2.8: New features for data integration and network visualization. Bioinformatics 27: 431-432, 2011.

19. Takeda K, Kaisho T and Akira S: Toll-like receptors. Ann Rev Immunol 21: 335-376, 2003.

20. GantnerBN,Simmons RM, Canavera SJ, Akira S and Underhill DM: Collaborative induction of inflammatory responses by dectin-1 and Toll-like receptor 2. J Exp Med 197: 1107-1117, 2003.

21. Netea MG, Van der Graaf CA, Vonk AG, Verschueren I, Van der Meer JW and Kullberg BJ: The role of toll-like receptor (TLR) 2 and TLR4 in the host defense against disseminated candidiasis. J Infect Dis 185: 1483-1489, 2002.

22. Zakikhany K, Naglik JR, Schmidt-Westhausen A, Holland G, Schaller $M$ and Hube B: In vivo transcript profiling of Candida albicans identifies a gene essential for interepithelial dissemination. Cell Microbiol 9: 2938-2954, 2007.

23. Weindl G, Naglik JR, Kaesler S, Biedermann T, Hube B, Korting HC and Schaller M: Human epithelial cells establish direct antifungal defense through TLR4-mediated signaling. J Clin Invest 117: 3664-3672, 2007.

24. Gow NA and Hube B: Importance of the Candida albicans cell wall during commensalism and infection. Curr Opin Microbiol 15: 406-412, 2012.

25. Moyes DL, Murciano C, Runglall M, Islam A, Thavaraj S and Naglik JR: Candida albicans yeast and hyphae are discriminated by MAPK signaling in vaginal epithelial cells. PLoS One 6: e26580, 2011.

26. Dongari-Bagtzoglou A and Fidel P Jr: The host cytokine responses and protective immunity in oropharyngeal candidiasis. J Dent Res 84: 966-977, 2005.

27. Mostefaoui Y,BartC,FrenetteMandRouabhia M: Candidaalbicans and Streptococcus salivarius modulate IL-6, IL-8 and TNF-alpha expression and secretion by engineered human oral mucosa cells. Cell Microbiol 6: 1085-1096, 2004.

28. Kempe S, Kestler H, Lasar A and Wirth T: NF-кB controls the global pro-inflammatory response in endothelial cells: evidence for the regulation of a pro-atherogenic program. Nucleic Acids Res 33: 5308-5319, 2005

29. Herrero de Dios C, Román E, Monge RA and Pla J: The role of MAPK signal transduction pathways in the response to oxidative stress in the fungal pathogen Candida albicans: implications in virulence. Curr Protein Pept Sci 11: 693-703, 2010.

30. Vallabhapurapu $S$ and Karin M: Regulation and function of NF- $\kappa B$ transcription factors in the immune system. Annu Rev Immunol 27: 693-733, 2009.

31. Yumoto H, Nakae H, Fujinaka K, Ebisu $\mathrm{S}$ and Matsuo T: Interleukin-6 (IL-6) and IL-8 are induced in human oral epithelial cells in response to exposure to periodontopathic Eikenella corrodens. Infect Immun 67: 384-394, 1999. 